\title{
Effects of the Short-Range Exchange Interaction on Plasmon Modes of Monolayer Black Phosphorous
}

\author{
T. Vazifehshenas ${ }^{1}$, S. Saberi-Pouya ${ }^{1,2}$ \\ ${ }^{1}$ Department of Physics, Shahid Beheshti University \\ G. C., Evin, Tehran 1983969411, Iran \\ t-vazifeh@sbu.ac.ir; s_saberi@sbu.ac.ir \\ ${ }^{2}$ Department of Physics, University of Antwerp \\ Groenenborgerlaan 171, B-2020 Antwerpen, Belgium
}

\begin{abstract}
We theoretically study the anisotropic collective plasmon excitations of monolayer black phosphorus beyond the random phase approximation by considering the short-range exchange interaction between the electrons. By applying the Hubbard local field correction to the dielectric function, we calculate the plasmon dispersion relation and loss function at room temperature. Numerical results show that the plasmon mode energies decrease along both main crystallographic directions when the Hubbard local field correction factor is taken into account. This effect is slightly stronger in the direction of lighter mass. Furthermore, we investigate the effect of local field on the loss function at different electron densities and obtain that the shift in plasmon frequency with density variation is smaller when the exchange interaction is included. Our findings may be useful for future studies on phosphorene-based nano-optic and plasmonic devices.
\end{abstract}

Keywords: Monolayer Black Phosphorus, Phosphorene, Plasmon, Anisotropy, Local Field Correction, Nano-Optics.

\section{Introduction}

Newly discovered two-dimensional (2D) materials, such as graphene [1], monolayer and few-layer black phosphorous [2], transition-metal dichalcogenides (TMDs) [3] and hexagonal boron-nitride [4] have provided a wide range of different novel properties and various potential applications. Black phosphorus which is the most thermodynamically stable phase of phosphorus at ambient temperature and pressure [5,6] consists of puckered hexagonal layers weakly coupled through the van der Waals interlayer interactions [7,8]. Phosphorene, a monolayer of black phosphorous, with a direct energy gap [9] and high carrier mobility [10] has recently attracted significant attention as a new 2D semiconductor material [11]. A special characteristic of phosphorene is the high in-plane anisotropy of its band structure. This anisotropy comes from the layered puckered-honeycomb structure of phosphorene resulting from the $s p^{3}$ hybridization. Motivated by this peculiar feature, several theoretical and experimental studies have investigated different anisotropic properties of phosphorene $[12,13]$. The crystallography-dependent properties of phosphorene make its electronic behavior more controllable. Recent studies reveal a high degree of anisotropic electrical and optical properties of phosphorene, which further confirm the importance of this new 2D semiconductor as a promising candidate for nano-electronic [10,14], nano-thermoelectric [15] and plasmonic applications [16,17].

Plasmon resonances which are the collective oscillations of electron density have a profound influence on the properties of metals and doped semiconductors. The dispersion relation of plasmons can be obtained by looking for the zeros of the dynamical dielectric function of the system. Recently, the many-body aspects of phosphorene have been also addressed through the study of highly anisotropic collective excitation modes in the doped monolayer and Coulomb coupled few-layer black phosphorus [18-22]. In these nanosheets, the direction-dependent plasmon resonances are located in the mid-infrared region of the electromagnetic spectrum which make phosphorene a good potential candidate for the molecular sensing and nano-optics. Also, the system anisotropy can be used as a means to restrict or permit the propagation of plasmons [11] which is important for nano-photonics application.

In this work, we calculate the plasmon modes in n-type doped phosphorene by going beyond the random phase approximation (RPA) where the short-range exchange interaction is taken into account. By including the Hubbard-type local field correction (LFC) in the dielectric function, we calculate the effect of exchange interaction on the anisotropic 
plasmon dispersion and loss function of monolayer black phosphorous at room temperature. It provides more reliable calculated results which may be useful for further investigations on the anisotropic nanostructures, specially in the low electron density limit.

The outline of this paper is as follows. In section 2, we briefly describe our formalism for computing the dielectric function of phosphorene monolayer. In the next section, we present our numerical calculations and discuss the results for the plasmon dispersion relation and loss function. Finally in section 4, we summarize our conclusion.

\section{Temperature-Dependent Dynamical Dielectric Function}

We consider a 2D electron gas system with an electron sheet density $n$ and an anisotropic parabolic-like band structure

$$
E_{\mathbf{k}}=\frac{\hbar^{2}}{2}\left(\frac{k_{x}^{2}}{m_{x}}+\frac{k_{y}^{2}}{m_{y}}\right)-\mu
$$

Here, $\mathbf{k}=\left(k_{x}, k_{y}\right)$ is a $2 \mathrm{D}$ wave vector, $m_{x}$ and $m_{y}$ are the diagonal elements of mass tensor, $\widehat{M}$, along $x$ and $y$ directions and $\mu$ is the chemical potential which is determined by the particle number conservation condition [23]. The dynamical dielectric function, $\varepsilon(\mathbf{q}, \omega)$, at finite temperature within the RPA is given by:

$$
\varepsilon(\mathbf{q}, \omega)=1-v(q) \Pi(\mathbf{q}, \omega)
$$

where $\omega$ is the frequency and $\nu(q)=2 \pi e^{2} / \kappa q$ is the unscreened 2D Coulomb interaction with $\kappa$ being the average dielectric constant. The non-interacting polarization function, $\Pi(\mathbf{q}, \omega)$, is obtained from the following equation [24]:

$$
\Pi(\mathbf{q}, \omega)=\frac{g_{s}}{A} \sum_{\mathbf{K}} \frac{f^{0}\left(E_{\mathbf{q}}\right)-f^{0}\left(E_{\mathbf{k}+\mathbf{q}}\right)}{E_{\mathbf{q}}-E_{\mathbf{k}+\mathbf{q}}+\hbar \omega+i \eta}
$$

Here, $f^{0}\left(E_{\mathbf{q}}\right)$ is the Fermi distribution function at energy $E$ corresponding to the wave vector $\mathbf{q}, g_{s}$ is spin degeneracy, $A$ is the area of system and $\eta$ is the broadening parameter, which accounts for disorder in the system. The temperaturedependent dynamic polarization function for a 2D material with anisotropic parabolic energy dispersion is given by [21]:

$$
\frac{\Pi(\mathbf{q}, \omega)}{g_{2 d}}=-\int d K \frac{\Phi(K, T)}{Q}\left[\operatorname{sgn}\left(\mathfrak{R}\left(Z_{-}\right)\right) \frac{1}{\sqrt{Z_{-}^{2}-K^{2}}}-\operatorname{sgn}\left(\mathfrak{R}\left(Z_{+}\right)\right) \frac{1}{\sqrt{Z_{+}^{2}-K^{2}}}\right]
$$

In the above symmetric form of temperature-dependent anisotropic polarization function, we define $\mathbf{Q}=\sqrt{m_{d} / \widehat{M}}\left(\mathbf{q} / k_{F}\right)$, $\mathbf{K}=\sqrt{m_{d} / \widehat{M}}\left(\mathbf{k} / k_{F}\right)$ with $k_{F}=\sqrt{2 \pi n}$ and $m_{d}=\sqrt{m_{x} m_{y}}$ is the 2D density of state mass. $\operatorname{Moreover}, \operatorname{sgn}(\mathfrak{R}(z))$ stands for the sign of real part of $z$ and we have $g_{2 d}=m_{d} / \pi \hbar^{2}, Z_{ \pm}=\left[(\hbar \omega+i \eta) / \hbar Q v_{F}\right] \pm(Q / 2)$ with $v_{F}=$ $\hbar k_{F} / m_{d}$ and $\Phi(K, T)=K /\left(1+\exp \left[\left(K^{2} E_{F}-\mu\right) / k_{B} T\right]\right)$. Here, $E_{F}, k_{B}$ and $T$ are the Fermi energy, Boltzmann constant and temperature, respectively and we consider $\mathbf{q}=q(\cos \theta, \sin \theta)$, in accordance to the notation in Ref. [20]. In case of phosphorene, we set $m_{x} \approx 0.15 m_{0}$ and $m_{x} \approx 0.7 m_{0}$ where $m_{0}$ is the free electron mass [17]. As it is well known, the electronic collective modes are obtained from zeros of the dielectric function, Eq. (2).

For systems with high electron density, the results of RPA calculations are rather reliable [25]. However, in the low electron density limit, the short-range interactions can not be ignored and must be included in the dielectric function by replacing $(1-G(q)) v(q)$ for $v(q)$ where $G(q)$ denotes the static LFC factor. Here, we incorporate only the exchange interaction and use the Hubbard-type LFC factor [26]: 


$$
G(q)=\frac{q}{2 \sqrt{q^{2}+k_{F}^{2}}}
$$

Employing the above relations, we can calculate the plasmon dispersion and loss function in the presence of exchange interaction.

\subsection{Plasmons and Energy Loss Function}

In this section, we study the plasmon collective modes obtained from zeros of the local field corrected dielectric function, $\varepsilon(\mathbf{q}, \omega)$, for the n-type doped monolayer black phosphorus at $T=300 \mathrm{~K}$. These modes correspond to the poles in the energy loss function which is proportional to the imaginary part of the inverse dielectric function, $|\mathfrak{J}(1 / \varepsilon(\mathbf{q}, \omega))|$, and can be measured by means of the electron energy loss spectroscopy. To make the anisotropic nature of phosphorene clear, we show the plasmon dispersion and loss function for two main crystallographic directions: $\theta=0(q \| x)$ and $\theta=\pi / 2(q \| y)$. The plasmon dispersion relation of phosphorene calculated from the Hubbard dielectric function is depicted in Fig. 1 at an electron density of $1 \times 10^{13} \mathrm{~cm}^{-2}$. For comparison, the results of the RPA calculations are also plotted. The density parameter, which is defined as the average distance between electrons in a non-interacting 2D electron gas, $r_{s}=$ $\sqrt{2} /\left(k_{F} a_{B}^{*}\right)$ with effective Bohr radius $a_{B}^{*}=\kappa /\left(e^{2} m_{d}\right)$ gives a measure for reliability of the RPA. In this figure, we choose $n=1 \times 10^{13} \mathrm{~cm}^{-2}$ and $\mathrm{SiO}_{2}$ as the substrate (with $\kappa \approx 2.5$ ) that yields $r_{S} \approx 4$.

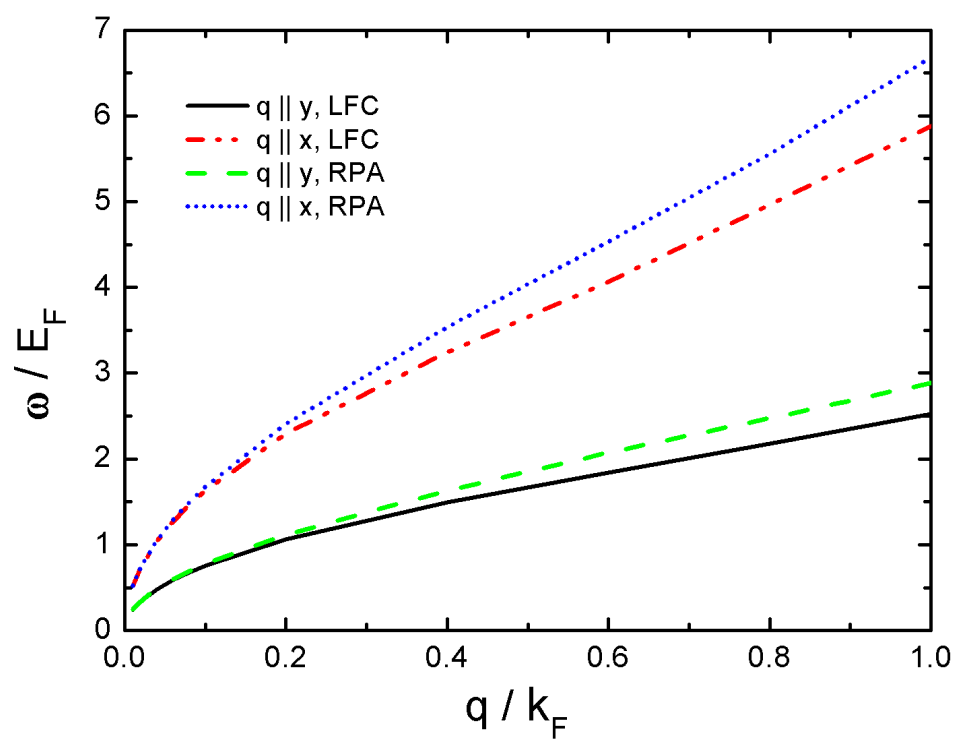

Fig. 1: Plasmon dispersion of black phosphorous monolayer on the $\mathrm{SiO}_{2}$ substrate along two main crystallographic directions: $\theta=0$ $(q \| x)$ and $\theta=\pi / 2(q \| y)$ at $T=300 \mathrm{~K}$ and $n=1 \times 10^{13} \mathrm{~cm}^{-2}$ with $\eta=1 \mathrm{meV}$.

It can be observed that including the exchange interaction apparently reduces the plasmon energies along both $x$ and $y$ directions and has the slightly stronger effect on the plasmon modes along the $x$ direction. This reduction is a consequence of increasing the screening effect, thus the electrons move more freely and the plasma oscillations can be excited more easily. Our numerical results for the loss function at small $q$ are shown in Fig. 2. 


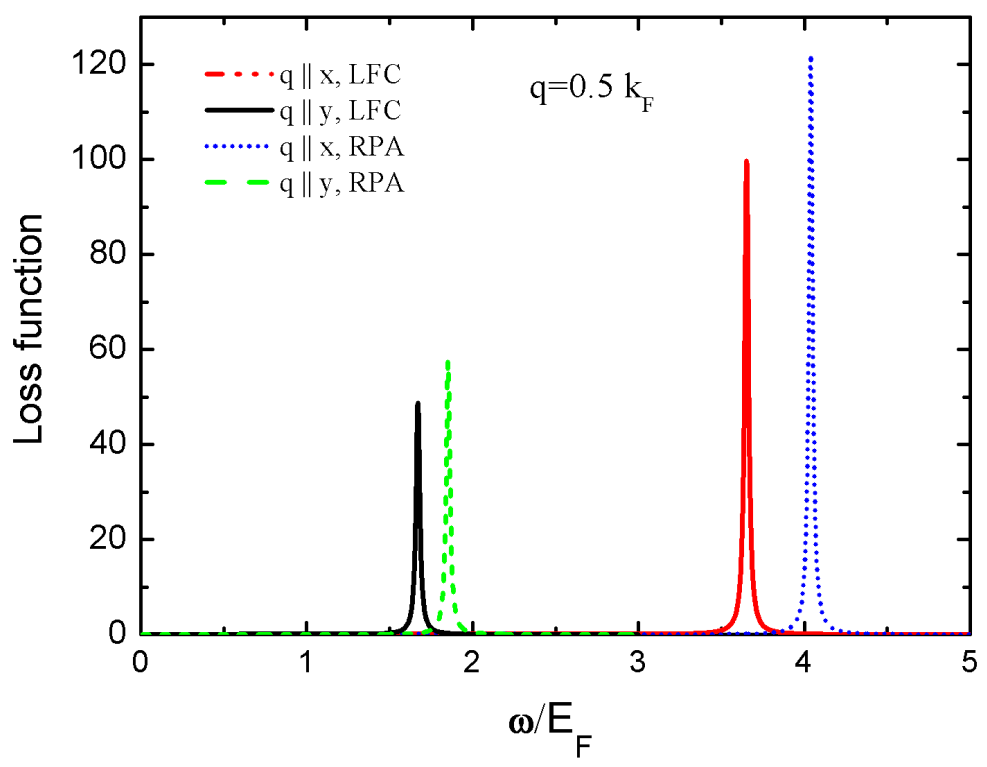

Fig. 2: Loss function of black phosphorous monolayer, $|\mathfrak{I}(1 / \varepsilon(\mathbf{q}, \omega))|$, along two main crystallographic directions: $\theta=0(q \| x)$ and $\theta=\pi / 2(q \| y)$ at $T=300 \mathrm{~K}$ and $n=1 \times 10^{13} \mathrm{~cm}^{-2}$ with $\eta=1 \mathrm{meV}$.

As expected, the LFC moves the peak of loss function to lower frequencies and this shift is more pronounced along the lighter mass $(x)$ direction. Finally, in Fig. 3, we show the effect of changing electron density on the loss function of phosphorene calculated from the Hubbard approximation along both $x$ and $y$ directions and compare with the RPA calculations. One can see that the blue shift of plasmon modes with decreasing the electron density obtained from the LFC is smaller than that of the RPA along both $x$ and $y$ directions.

\section{Conclusion}

To summarize, we have investigated the effect of Hubbard exchange interaction on the anisotropic plasmon dispersion and loss function of black phosphorous monolayer. Using the temperature-dependent dynamical polarization function of phosphorene and Hubbard static LFC factor, we have calculated the plasmon modes at room temperature. The results show that the inclusion of LFC results in a slightly larger reduction of the plasmon energies along the $x$ direction. Moreover, we have studied the effects of LFC on the anisotropic loss function for two different electron densities and obtained that the LFC calculations predict a smaller shift of plasmons by changing the electron density. Since the anisotropic plasmon modes are differently affected by the short-range exchange interaction, this allows a promising possibility to probe the direction-dependent many-body effects in a nanosheet with anisotropic band structure. Also, our results can be helpful for highly anisotropic molecular sensing, nano-optic and plasmonic applications. 

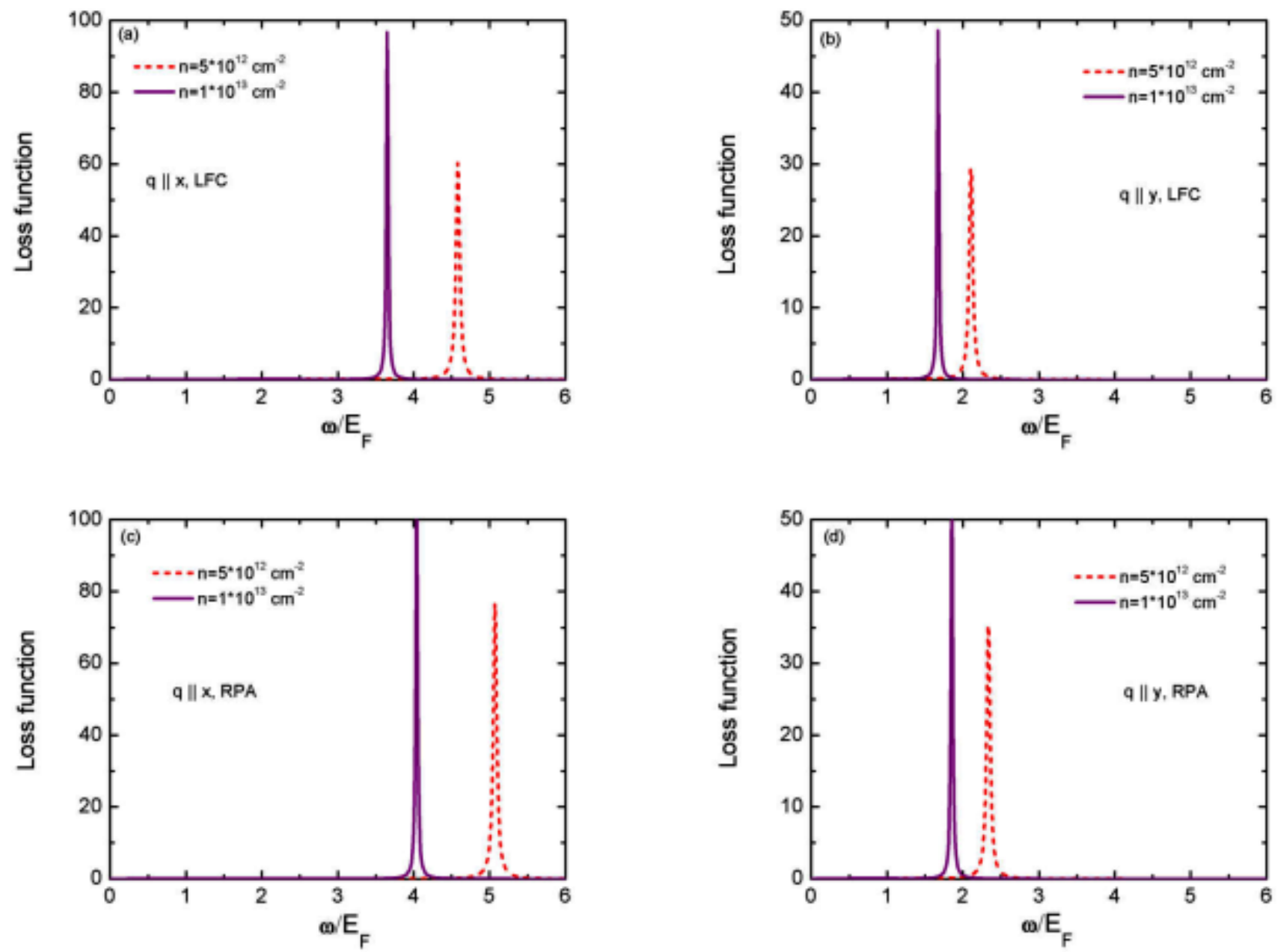

Fig. 3: Effect of changing density on loss function, $|\Im(1 / \varepsilon(\mathbf{q}, \omega))|$, (a),(c) for $\theta=0(q \| x)$ and (b),(d) for $\theta=\pi / 2(q \| y)$. (a),(b) calculated within LFC approximation and (c),(d) calculated within RPA. Here, we use two different electron densities $n=5 \times$ $10^{12} \mathrm{~cm}^{-2}$ and $1 \times 10^{13} \mathrm{~cm}^{-2}$ at $q=0.5 k_{F}$ with $\eta=1 \mathrm{meV}$.

\section{References}

[1] A. K. Geim and I. V. Grigorieva, "Van der Waals heterostructures," Nat., vol. 499, pp. 419-425, 2013.

[2] X. Ling, H. Wang, S. Huang, F. Xia and M. S. Dresselhaus, "The renaissance of black phosphorus," Proc. Natl. Acad. Sci. U.S.A., vol. 112, no. 15, pp. 4523-4530, 2015.

[3] G.-B. Liu, D. Xiao, Y. Yao, X. Xu and W. Yao, "Electronic structures and theoretical modelling of two-dimensional group-VIB transition metal dichalcogenides," Chem. Soc. Rev., vol. 44, no. 9, pp. 2643-2663, 2015.

[4] M. Xu, T. Liang, M. Shi and H. Chen, "Graphene-like two-dimensional materials," Chem. Rev., vol. 113, no. 5, pp. 3766-3798, 2013.

[5] R. W. Keyes, "The electrical properties of black phosphorus," Phys. Rev., vol. 92, no. 3, pp. 580-584, 1953.

[6] J. C. Jamieson, "Crystal structures adopted by black phosphorus at high pressures," Science, vol. 139, pp. 12911292, 1963.

[7] S. Appalakondaiah, G. Vaitheeswaran, S. Lebègue, N. E. Christensen and A. Svane, "Effect of van der Waals interactions on the structural and elastic properties of black phosphorus," Phys. Rev. B, vol. 86, no. 3, pp. 035105(9), 2012.

[8] L. Li and et al, "Direct observation of the layer-dependent electronic structure in phosphorene," Nat. Nanotechnol., vol. 12, 21-25, 2017.

[9] V. Tran, R. Soklaski, Y. Liang and L. Yang, "Layer-controlled band gap and anisotropic excitons in few-layer black phosphorus," Phys. Rev. B, vol. 89, no. 23, pp. 235319(6), 2014. 
[10] H. Liu, A. T. Neal, Z. Zhu, Z. Luo, X. Xu, D. Tománek and P. D. Ye, "Phosphorene: an unexplored 2D semiconductor with a high hole mobility," ACS nano, vol. 8, no. 4, pp. 4033-4041, 2014.

[11] N. Carvalho, M. Wang, X. Zhu, A. S. Rodin, H. Su and A. H. C. Neto, "Phosphorene: from theory to applications," Nature Reviews Materials, vol. 1, pp. 16061(16), 2016.

[12] S. Saberi-Pouya, T. Vazifehshenas, T. Salavati-fard, M. Farmanbar and F. M. Peeters, "Strong anisotropic optical conductivity in two-dimensional puckered structures: The role of the Rashba effect," Phys. Rev. B, vol. 96, no. 6, pp. 075411(7), 2017.

[13] J. Qiao, X. Kong, Z.-X. Hu, F. Yang and W. Ji, "Rediscovering black phosphorus as an anisotropic layered material for optoelectronics and electronics," Nat. Commun., vol. 5, pp. 4458, 2014.

[14] B. Sa, Y.-L. Li, Z. Sun, J. Qi, C. Wen and B. Wu, "The electronic origin of shear-induced direct to indirect gap transition and anisotropy diminution in phosphorene," Nanotechnology, vol. 26, no. 21, pp. 215205, 2015.

[15] T.-H. Liu and C.-C. Chang, "Anisotropic thermal transport in phosphorene: effects of crystal orientation," Nanoscale, vol. 7, no.11, pp. 10648-10654, 2015.

[16] A. N. Rudenko and M. I. Katsnelson, "Quasiparticle band structure and tight-binding model for single- and bilayer black phosphorus," Phys. Rev. B., vol. 89, no. 20, pp. 201408(5), 2014.

[17] T. Low, A. S. Rodin, A. Carvalho, Y. Jiang, H. Wang, F. Xia and A. H. Castro Neto, "Tunable optical properties of multilayer black phosphorus thin films," Phys. Rev. B, vol. 90, no. 7, pp. 075434(5), 2014.

[18] D. A. Prishchenko, V. G. Mazurenko, M. I. Katsnelson and A. N. Rudenko, "Coulomb interactions and screening effects in few-layer black phosphorus: a tight-binding consideration beyond the long-wavelength limit," $2 D$ Materials, vol. 4, no. 2, pp. 025064, 2017.

[19] T. Low, R. Roldán, H. Wang, F. Xia, P. Avouris, L. M. Moreno and F. Guinea, "Plasmons and screening in monolayer and multilayer black phosphorus," Phys. Rev. Lett., vol. 113, no.10, pp. 106802(5), 2014.

[20] A. S. Rodin and A. H. Castro Neto, "Collective modes in anisotropic double-layer systems," Phys. Rev. B, vol. 91, no. 7 , pp. 075422(5), 2015.

[21] S. Saberi-Pouya, T. Vazifehshenas, M. Farmanbar and T. Salavati-fard, "Coulomb drag in anisotropic systems: a theoretical study on a double-layer phosphorene," Journal of Physics: Condensed Matter, vol. 28, no. 28, pp. 285301, 2016.

[22] S. Saberi-Pouya, T. Vazifehshenas, T. Salavati-fard and M. Farmanbar, "Anisotropic hybrid excitation modes in monolayer and double-layer phosphorene on polar substrates," Phys. Rev. B, vol. 96, no. 11, p. 115402(9), 2017.

[23] N. Ashcroft and N. Mermin, Solid State Physics. Saunders College, Philadelphia, 1976.

[24] D. Bohm and D. Pines, "A collective description of electron interactions: III. Coulomb interactions in a degenerate electron gas," Phys. Rev., vol. 92, no. 3, pp. 609-625, 1953.

[25] E. H. Hwang and S. Das Sarma, "Plasmon modes of spatially separated double-layer graphene," Phys. Rev. B, vol. 80, no. 20, pp. 205405(5), 2009.

[26] M. Jonson, "Electron correlations in inversion layers," J. Phys. C: Sol. State Phys., vol. 9, no. 16, pp. $3055,1976$. 